\title{
Fatigue Life Prediction of Self-Piercing Rivet Joints Between Magnesium and Aluminum Alloys
}

\author{
Hong-Tae Kang ${ }^{1, *}$ and Sai Boorgu ${ }^{1}$ \\ ${ }^{1}$ The University of Michigan-Dearborn, 4901 Evergreen Road, Dearborn, MI, USA
}

\begin{abstract}
Various light materials including aluminum alloys and magnesium alloys are being used to reduce the weight of vehicle structures. Joining of dissimilar materials is always a challenging task to construct a solid structure. Self-piercing rivet (SPR) joint is one of various joining methods for dissimilar materials. Front shock tower structures were constructed with magnesium alloy (AM60) joined to aluminum alloy (Al6082) by SPR joints. To evaluate the durability performance of the SPR joints in the structures, fatigue tests of the front shock tower structures were conducted with constant amplitude loadings. Furthermore, this study investigated fatigue life prediction method of SPR joints and compared the fatigue life prediction results with that of experimental results. For fatigue life prediction of the SPR joints in the front shock tower structures, lap-shear and cross-tension specimens of SPR joint were constructed and tested to characterize the fatigue properties of the SPR joint. Then, the SPR joint was represented with area contact method (ACM) in finite element (FE) models. The load-life curves of the lap-shear and cross-tension specimens were converted to a structural stress-life (S-N) curve of the SPR joints. The S-N curve was used to predict fatigue life of SPR joints in the front shock tower structures. The test results and the prediction results were well correlated.
\end{abstract}

\section{Introduction}

Automotive industries continue to develop fuel-efficient vehicles by reducing their weight. Magnesium $(\mathrm{Mg})$ and Aluminum ( $\mathrm{Al})$ alloys are a viable option for the weight reduction of vehicle structures due to their low density. Currently automotive companies are producing various vehicle structures with aluminum alloys. Many efforts are also focusing on developing vehicle structures and components with magnesium alloys. Furthermore, some researchers demonstrated for constructing vehicle structures and components with dissimilar lightweight materials to reduce the weight of vehicles.

Joining technology plays key role to construct vehicle structures with dissimilar light materials including aluminum alloys and magnesium alloys. The self-piercing rivet (SPR) is a widely employed mechanical joining technology ductile materials [7]. This joining method is similar to the traditional riveting but without a pre-drilling procedure. Thus, SPR technology can achieve rapid assembly time and high joint integrity. Many vehicle manufacturers replaced the traditional electrical resistance spot welds to SPR joints to join $\mathrm{Al} / \mathrm{Al}$ and $\mathrm{Al} /$ steel. Some researchers also demonstrated the employment of SPR for joining magnesium alloys with aluminum alloys.

In this research front shock tower structures of a passenger vehicle were constructed with magnesium alloy
(AM60), aluminum alloys (A16082) and sheet steel (HSLA350). The main part of the shock tower is made of AM60 and the upper rail and the lower rail of the structures are made of HSLA350 and Al6082, respectively. AM60 and HSLA350 are joined with adaptable insert weld (AIW) that is a hybrid joining technique of electrical resistance spot welding and riveting. AM60 and Al6082 are joined with SPR.

Durability tests of the constructed structures were conducted to evaluate the structural performance of the joints and the base material of the shock tower components. The loading direction applied for the durability tests was determined to evaluate a specific joint or base material. X-direction loading as shown in Figure 1 was intended to assess durability of SPR joints between AM60 and Al6082. To evaluate AIW joints, Y-direction loading was applied to the loading fixture as shown in Figure 1. The test results and prediction activities were reported in [1]. The fatigue performance for the base material of AM60 was assessed by applying load in Zdirection as shown in Figure 1. The test results and fatigue life prediction activities were reported in [2].

This study reports a fatigue life prediction method for SPR joints between AM60 and A16082 and validate the method using front shock tower structures. To achieve the goal, lap-shear (LS) and cross-tension (CT) specimens were fabricated and tested with quasi-static loading and constant amplitude cyclic loading. The fatigue test results

\footnotetext{
* Corresponding author: htkang@umich.edu
} 
of LS and CT specimens were correlated with structural stress amplitude of SPR joints. This relationship between the structural stress and fatigue life becomes the joint fatigue property of SPR joints for AM60 and Al6082. Then, this joint fatigue property is used to predicted fatigue life of SPR joints in the front shock tower structures. Finally, the prediction results and test results were compared to ensure the effectiveness of the method used in this study.

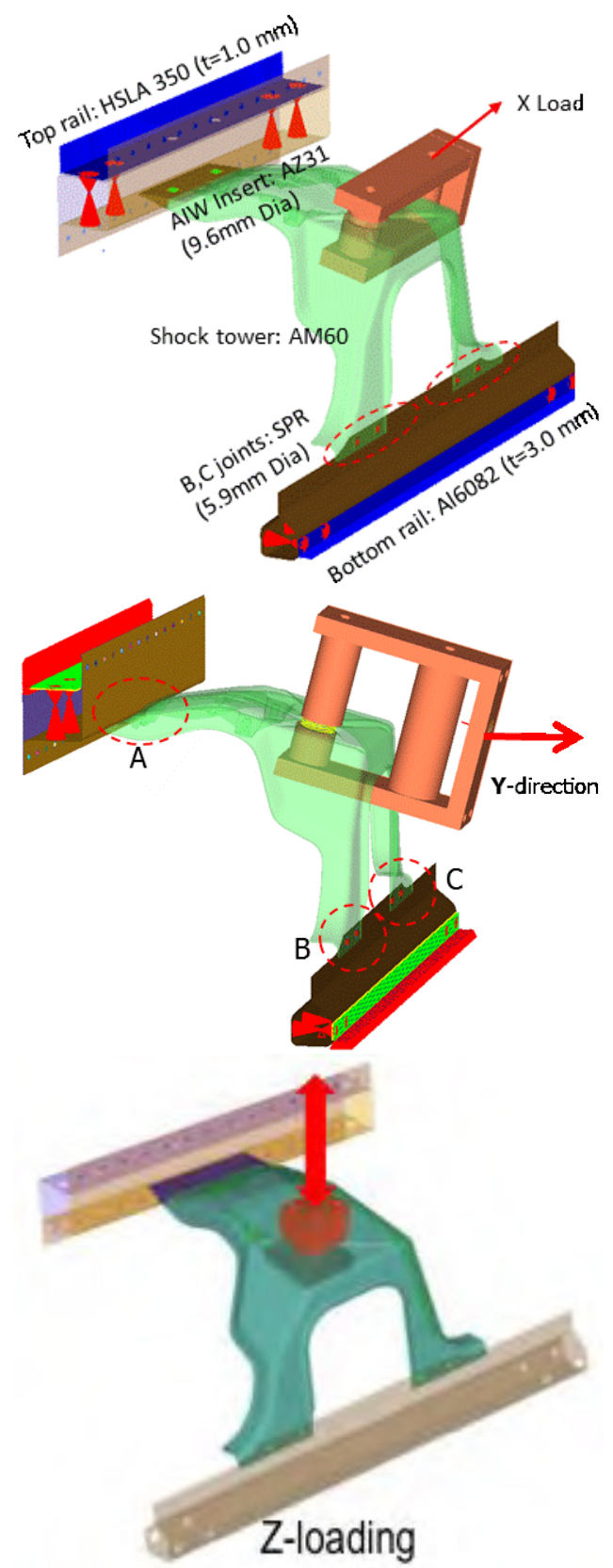

Fig. 1. Fatigue loading directions for the front shock tower structures.

\section{Specimen Geometry and Dimensions}

Specimens of lap-shear and cross-tension were used to characterize the performance of SPR joints in this study. The upper sheet was made of AM60 with thickness of 2.0 $\mathrm{mm}$ and lower sheet was Al6082 of $3.0 \mathrm{~mm}$ thick. The dimensions of the specimens are shown in Figure 2. The cross section of a typical SPR joint between AM60 and Al6082 is shown in Figure 3.
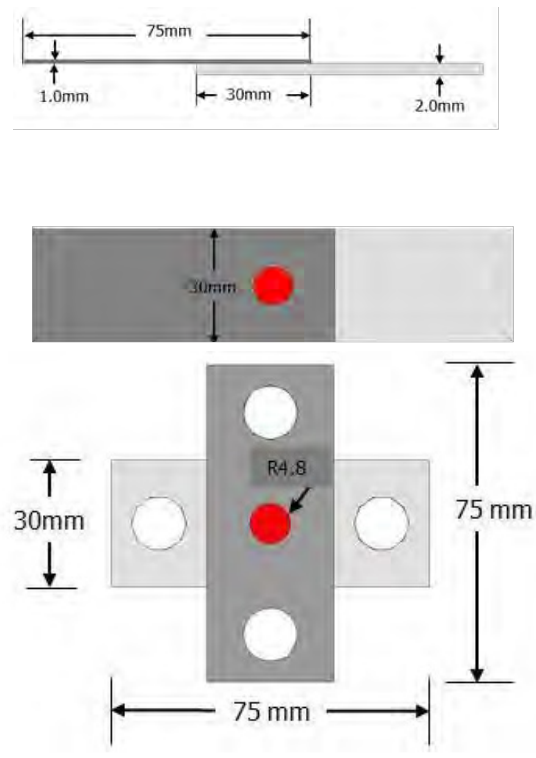

Fig. 2. Specimen geometry and dimension for the lap-shear and cross-tension specimen of SPR joint.

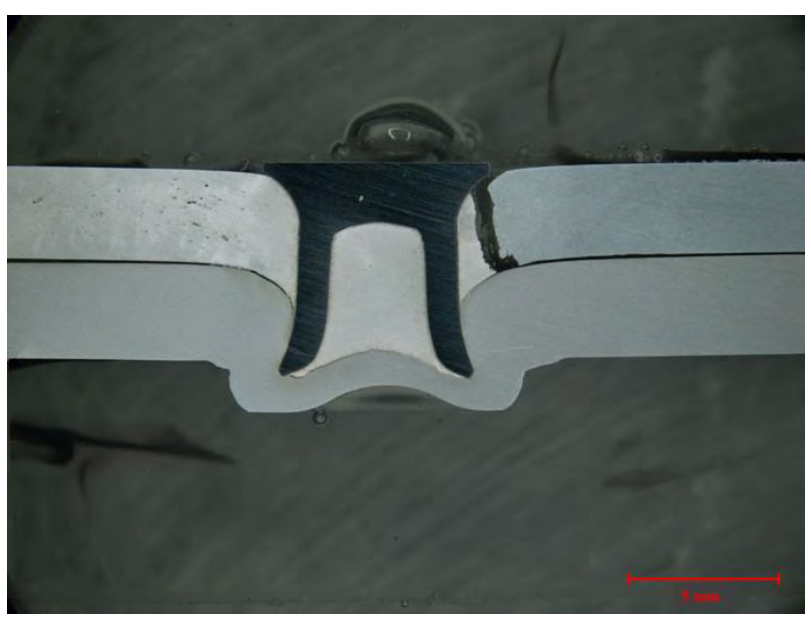

Fig. 3. A cross section of SPR joint between AM60 and Al6082.

\section{Fatigue Test Results of Coupons}

Fatigue tests for the specimens were conducted with constant amplitude loads at a load ratio $\left(\mathrm{R}=\left(\mathrm{P}_{\min } / \mathrm{P}_{\max }\right)\right.$ of 0.1 . These tests were performed at six different load levels for lap-shear specimens with two repetitions at each load level. For cross tension specimens fatigue tests were conducted at four different load levels with three repetitions at each load level. The load ranges for fatigue testing of specimens were determined from quasi-static tests of the specimens so that the fatigue life ranges between $1 \times 10^{3}$ and $5 \times 10^{6}$ cycles. The definition of fatigue failure was a complete separation of the specimen in two pieces. The typical failure mode observed in fatigue tests was cracked at the cast AM60 in both the specimen geometries as shown in Figure 6. For all these tests the 
runout was defined at $5 \times 10^{6}$ cycles. The applied maximum load versus the cycle to failure of the SPR joint specimens are plotted in Figure 4. The runouts are presented as hollow circle and rectangle in the Figure 4.

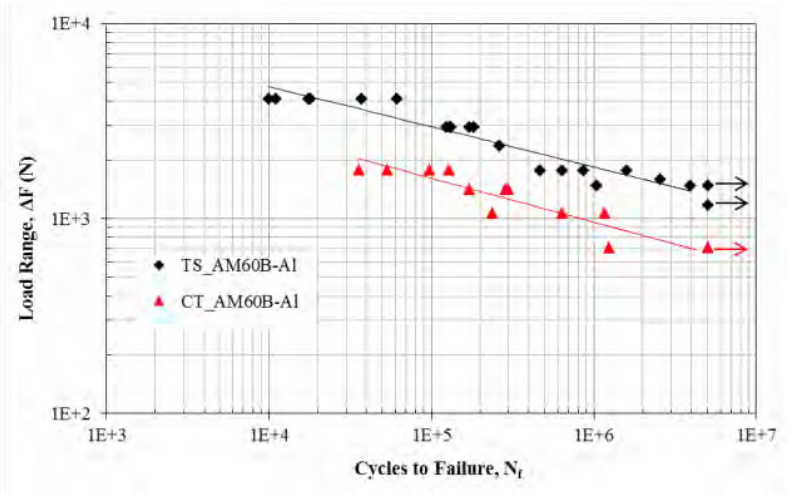

Fig. 4. Load range versus cycles to failure for the SPR specimens.

\section{Finite Element Representation of LS and CP Specimens}

The bottom sheet (A16082) was represented with shell elements along the mid plane of the sheet. The element size of the bottom sheet was $5 \mathrm{~mm}$, which is generally accepted element size for vehicle structures in automotive industries. The lower sheet (AM60) was modeled using three dimensional $2^{\text {nd }}$-order tetrahedral elements since AM60 in the shock tower structures is a casted component. Three dimensional $2^{\text {nd }}$-order tetrahedral elements are an easy and accurate way of representing the casting features in the shock tower structures. One strip of the LS and CP specimens was constrained in all-degrees of freedom and the other strip was constrained all-degrees of freedom except the loading direction of the specimen as constrain conditions were applied during the specimen testing. Figure 5 shows meshed finite element (FE) models of LS and CP specimens used in this study.
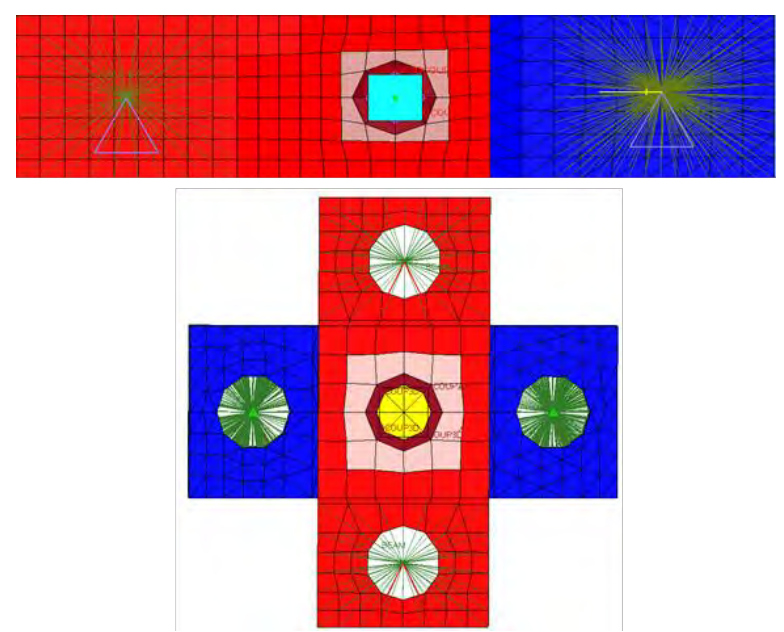

Fig. 5. Finite element models of LS and CP specimens. SPR joint in the specimens was represented with area contact method (ACM).
In this method the joint is represented using a solid hexahedral element with distributed coupling elements. The distributed coupling elements connect the nodes of the solid hexahedral element and the nodes of the top and bottom sheets as shown in Figure 5. The material properties of $\mathrm{AM} 60$ are $\mathrm{E}=44 \mathrm{GPa}, v=0.3$, and $\rho=1.6 \mathrm{E}-9$ tonne $/ \mathrm{mm}^{3}$. The material properties of Al6082 are $\mathrm{E}=70$ $\mathrm{GPa}, v=0.33$, and $\rho=1.6 \mathrm{E}-9$ tonne $/ \mathrm{mm}^{3}$.

\section{Structural Stress Calculation}

Structural stress is calculated from the forces and moments acting on the joint using Rupp and co-workers' approach [3]. The mechanics around SPR joint is not exactly the same with that of spot welded joint. However, the failure modes of SPR and spot welded joint are very similar. Thus, this study used the same structural stress equations developed for spot welded joints. This approach enables to avoid using fine meshed finite element model of SPR joints in vehicle body structures. This capability is so important in FE modeling of vehicle body structures that have many joints. Fine meshed representation for an SPR joint is only possible for a specimen level not for a structural level.

The stresses/strains obtained from FE analysis at the joints are not representing realistic ones because the joints are coarsely meshed. Thus, structural stresses are calculated at the joints using force and moment information less sensitive to the mesh density variations. The structural stress is, then used for constructing the Stress-Life (S-N) curve that defines fatigue property of SPR joints. The structural stress equations used in this study is briefly summarized below in Figure 6 [4].

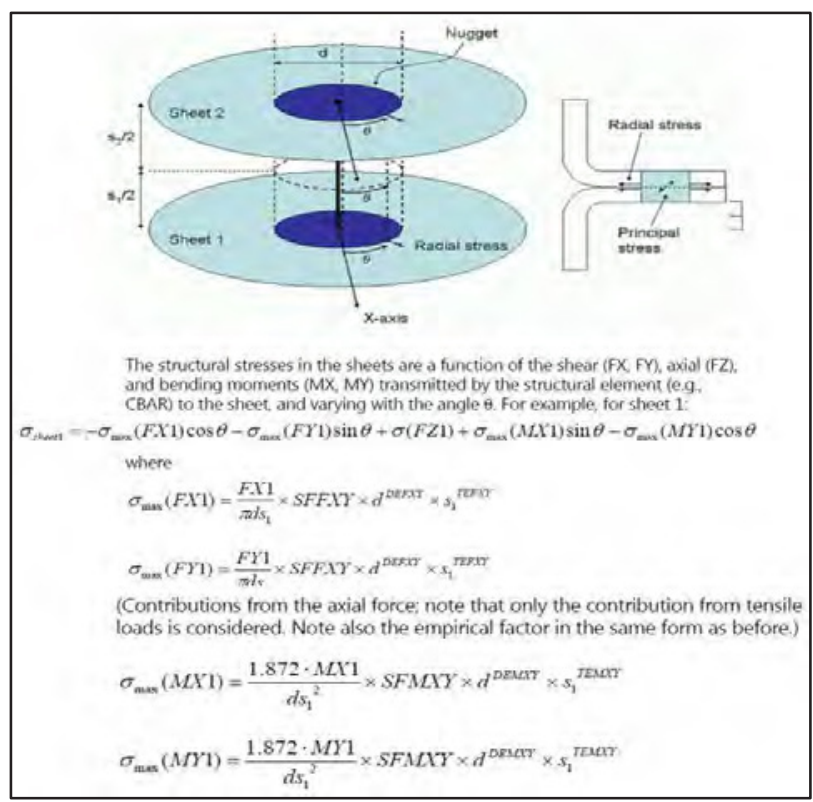

Fig. 6. Schematic drawing of spot weld presentation and structural stress equations [4].

To calculate structural stress, it is necessary to obtain the shear force components (FX1 and FY1), axial component 
(FZ1), and bending moment components (MX1 and MY1). It also requires empirical factors such as SFFXY, DEFXY, TEFXY, SFMXY, DEMXY, and TEMXY. These factors play an important role in collapsing structural stresses for different dimensions of sheet thickness, joint diameters and different joint geometries. These parameters can be determined by nonlinear optimization described in [5]. To construct S-N curve of SPR joints in this study, LS and CP specimens were fabricated and tested as described previous section. Also FE representations for those specimens were described. At each fatigue test load level for LS and CP specimens, forces and moments were obtained at the joint from FE analysis. The structural stress equations shown above were used to calculate the structural stress corresponding to the specific load level applied for fatigue testing. These structural stresses were then used to plot the structural stress range versus cycles to failure curve on a log-log scale as shown in Figure 7.

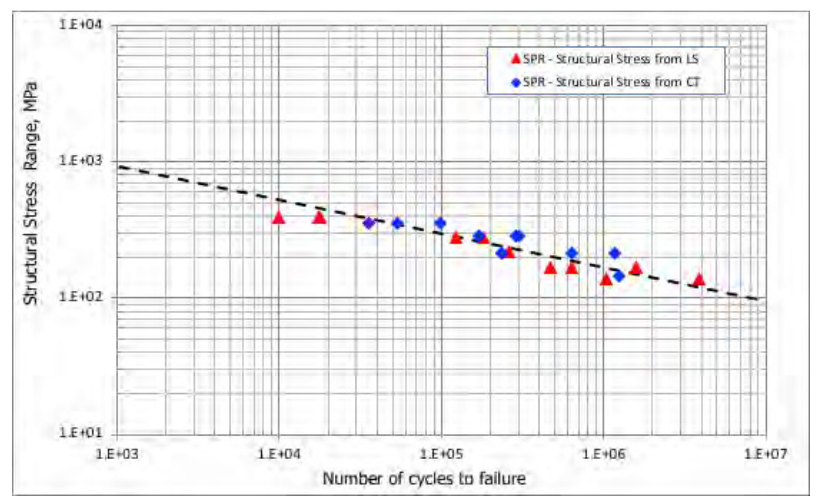

Fig. 7. S-N curve using structural stress ranges for LS and CP specimens.

\section{Testing of Front Shock Tower Structure}

Three quasi-static tests were conducted for the front shock tower structure subjected to X-direction loading as shown in Figure 1. The shock tower was constrained at the top and bottom rails by using fixtures and bolts. The load is applied using a jig that pulls the shock tower in $\mathrm{X}$ direction until failure is observed. During this ultimate strength testing the failure occurred at SPR and the average ultimate strength was 9,700 N. The failure was occurred at AM60 near a SPR joint location. The failure location of the ultimate strength testing is shown in Figure 8. This ultimate strength was then used to estimate the fatigue test load such that the result of fatigue life ranges between 1E3 to 1E6 cycles. Three load amplitude ranges were applied to investigate durability performance of the structures. A series of nine structures were tested in this study. As shown in Figure 8, all structures tested were failed at the SPR joint on left leg of the shock tower structure. Failure of the structure was defined as any visible crack is observed in the structure.
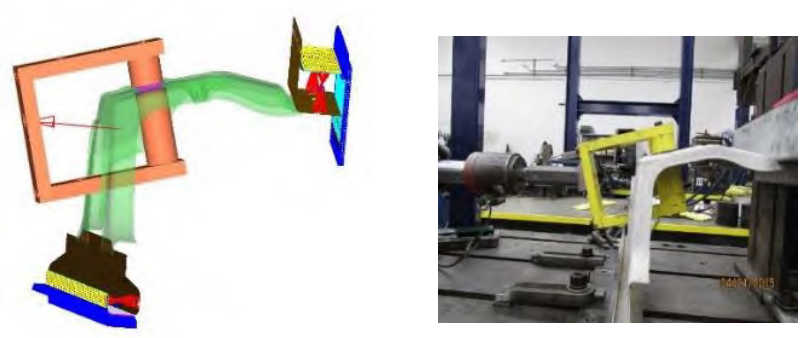

Fig. 8. Failure locations ofSPR joints subjected to quasi-static and fatigue loading.

\section{Fatigue Life Prediction of Joints in Shock Tower}

Before testing of the front chock tower structures, FE model of the structure was developed and analyzed under the boundary and loading conditions to identify the critical locations. The upper rail (HSLA350) and the lower rail (Al6082) were represented with shell elements and the shock tower (AM60) was represented with $2^{\text {nd }}$ order tetrahedral elements. The joints connecting the upper rail and the lower rail were presented with ACM as described in previous section.

The fatigue life prediction of SPR joints in the front shock tower structures was performed and compared with that of experimental results. The S-N curve obtained from LS and CP specimens of the SPR joint was used to predict fatigue life of SPR joints in the front shock tower structures. The FE prediction identified correctly the failure location of the SPR joint and fatigue life prediction results are within factor of 3 lines sown in Figure 9. The solid line represents the perfect correlation between the test results and prediction results. The upper and lower dotted lines represent the band for the factor of 3 .

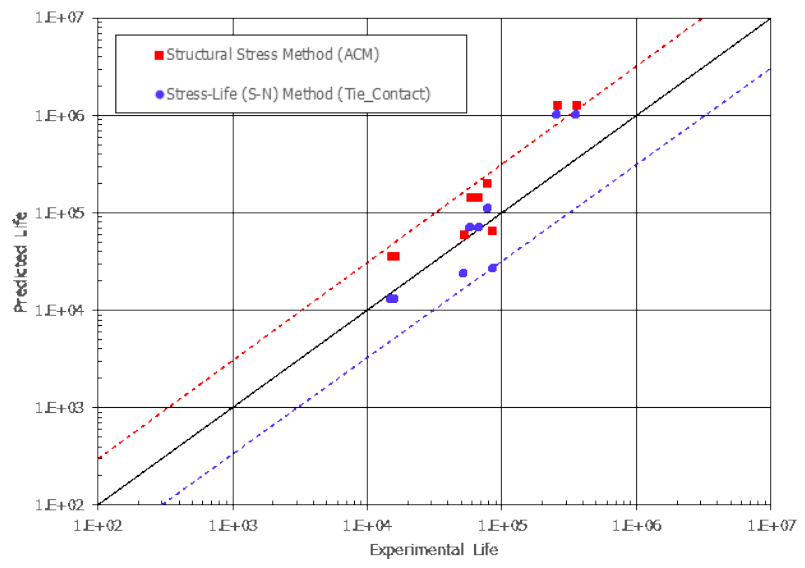

Fig. 9. Comparison between experimental results and prediction results for fatigue life of SPR joints in the front shock tower structures.

\section{Summary and Conclusions}

Lap shear and cross tension specimens were used to characterize the fatigue performance of SPR joints between AM60 and A16082. Based on the observation of the failure modes of the joints, it was assumed that the mechanics at the SPR joint are similar to those of the 
electrical resistance spot weld joint. Therefore, the same structural stress equations for spot welded joints were used for SPR joints between AM60 and Al6082. Fatigue test results of LS and CP specimen configurations were correlated well with a log linear function after the applied load ranges were converted to structural stress ranges. The S-N curve based on the structural stress ranges was then used to predict the fatigue life of SPR joints in the front shock tower structures. Fatigue test results of the front shock tower structures and their prediction results were compared. From this study, the following conclusions are made:

1. While the fatigue test results in load range versus life of LS and CT specimens were quite different, these differences are minimized after the test results were presented with structural stress range versus life for the LS and CP specimens.

2. Structural stress equations and methodology developed for electrical resistance spot welded joints are still applicable for SPR joints between AM60 and Al6082.

The authors would like to express thanks to A.K. Khosrovaneh (GM), X. Su (Ford), M. Guo (FCA), C. Jiang (AET), and Y. Lee (FCA) for their valuable helps for the data and discussions.

\section{References}

1. H. Kang, Z. Li, A.K. Khosrovaneh, X. Su, M. Guo, Y. Lee, and C. Jiang, SAE International Journal of Materials and Manufacturing, 8(3): 789-794, 2015.

2. H. Kang, A.K. Khosrovaneh, X. Su, M. Guo, Y. Lee, and C. Jiang, $73^{\text {rd }}$ IMA Conference, May 15-17, 2016, Rome, Italy.

3. Rupp, A., Storzel, K., and Grubisic, V., $S A E$ technical report No. 950711, Detroit, Michigan, 1995.

4. nCode, DesighLife User Manual, 2017.

5. Kang, H., Wu, X., Khosrovaneh, A.K., Li, Z., Fatigue and Fracture Test Planning, Test Data Acquisitions and Analysis, 2016, STP1598, pp. 198211. 